\title{
Vitamin D and cause-specific vascular disease and mortality: a Mendelian randomisation study involving 99,012 Chinese and 106,911 European adults
}

Tao Huang ${ }^{1,2+}$, Shoaib Afzal ${ }^{3 \dagger}$, Canqing Yu' ${ }^{1}$, Yu Guo ${ }^{4}$, Zheng Bian ${ }^{4}$, Ling Yang ${ }^{5}$, lona Y. Millwood ${ }^{5}$, Robin G. Walters ${ }^{5}$, Yiping Chen ${ }^{5}$, Ningyu Chen ${ }^{6}$, Ruqin Gao ${ }^{7}$, Junshi Chen ${ }^{8}$, Robert Clarke ${ }^{5}$, Zhengming Chen ${ }^{5}$, Christina Ellervik ${ }^{3,9,10}$, Børge G. Nordestgaard ${ }^{3,9,10}$, Jun LV $\mathrm{LV}^{1,2,11^{*}}$, Liming $\mathrm{Li}^{1,4^{*}}$ and on behalf of the China Kadoorie Biobank Collaborative Group

\begin{abstract}
Background: Randomised control trials and genetic analyses have demonstrated that vitamin D or 25-hydroxyvitamin $\mathrm{D}(25[\mathrm{OH}] \mathrm{D})$ levels may not play a causal role in the development of cardiovascular disease. However, it is unclear if $25(\mathrm{OH}) \mathrm{D}$ is causally associated with cause-specific vascular disease and lipids. Therefore, we examined the causal association of 25(OH)D with myocardial infarction, stroke, ischaemic heart disease, ischaemic stroke, subarachnoid haemorrhage, intracerebral haemorrhage, and lipid levels among both Chinese and Europeans.
\end{abstract}

Methods: We used a Mendelian randomisation (MR) design in the China Kadoorie Biobank, the Copenhagen City Heart Study, and the Copenhagen General Population Study. The 25(OH)D-related genetic variants in the CYP2R1 and DCHR7 genes were genotyped in 99,012 Chinese adults and 106,911 Danish adults.

Results: In Chinese adults, plasma 25(OH)D levels were not significantly associated with cause-specific vascular disease or mortality, with the exception of intracerebral haemorrhage ( $\mathrm{HR}, 1.09[95 \% \mathrm{Cl}, 1.01,1.18]$ per $25 \mathrm{nmol} / \mathrm{L}$ higher plasma 25(OH)D). In Europeans, plasma 25(OH)D levels were inversely associated with all types of vascular diseases and mortality. However, MR analysis did not demonstrate causal associations of genetically increased 25(OH)D levels with cause-specific vascular diseases, or mortality in both Chinese and European adults. In addition, each $25 \mathrm{nmol} / \mathrm{L}$ higher 25(OH)D was observationally associated with lower total cholesterol and low-density lipoprotein cholesterol levels, but higher high-density lipoprotein cholesterol levels. Likewise, MR analysis showed that 25(OH)D levels were not causally associated with lipids in both Chinese and European adults after Bonferroni correction.

Conclusions: We found no evidence to support that genetically increased 25(OH)D was associated with a lower risk of ischaemic stroke, intracerebral haemorrhage, subarachnoid haemorrhage, and lipid levels in both Chinese and European adults. These results suggest that the inverse associations of vitamin D with vascular disease could be the result of confounding.

Keywords: Mendelian randomisation, Vitamin D, Cardiovascular diseases, Lipids, Causal effect

\footnotetext{
* Correspondence: epi.lvjun@vip.163.com; Imlee@vip.163.com

${ }^{\dagger}$ Tao Huang and Shoaib Afzal contributed equally to this work.

'Department of Epidemiology and Biostatistics, School of Public Health,

Peking University Health Science Center, 38 Xueyuan Road, Beijing 100191,

China

Full list of author information is available at the end of the article
}

(c) The Author(s). 2019 Open Access This article is distributed under the terms of the Creative Commons Attribution 4.0 International License (http://creativecommons.org/licenses/by/4.0/), which permits unrestricted use, distribution, and reproduction in any medium, provided you give appropriate credit to the original author(s) and the source, provide a link to the Creative Commons license, and indicate if changes were made. The Creative Commons Public Domain Dedication waiver (http://creativecommons.org/publicdomain/zero/1.0/) applies to the data made available in this article, unless otherwise stated. 


\section{Background}

Observational studies have shown that low plasma levels of vitamin $\mathrm{D}$ are associated with higher risks of cardiovascular disease (CVD) [1-3] and mortality from CVD, cancer, and all causes $[2,4,5]$ in European populations. However, randomised intervention trials (RCTs) of vitamin D3 (cholecalciferol) supplementation have not clearly demonstrated any protective effect on CVD [6-9] or all-cause mortality $[10,11]$. Findings from Mendelian randomisation (MR) analysis also did not indicate causal association of 25hydroxyvitamin $\mathrm{D}(25[\mathrm{OH}] \mathrm{D})$, usually used to assess vitamin D status, with increased risk of ischaemic heart disease, myocardial infarction [12], and cardiovascular mortality [13], but showed a possible effect on all-cause and cancer mortality in Europeans [13].

No study has evaluated these associations in the Chinese population, while two studies examined risk of diabetes $[14,15]$. Importantly, the causal associations between $25(\mathrm{OH}) \mathrm{D}$ and cause-specific vascular disease such as stroke, ischaemic stroke, intracerebral haemorrhage, subarachnoid haemorrhage, and lipid levels have not been examined among European and Chinese adults. Whether a high $25(\mathrm{OH}) \mathrm{D}$ level is a cause of decreased vascular disease and mortality or simply a consequence of poor health is thus unclear. This question has important public health relevance, as about half of the population have vitamin $\mathrm{D}$ deficiency and regularly take vitamin D supplements [16], but without proper scientific evidence for any beneficial effect on many disease outcomes.

With the lack of evidence from intervention trials, a MR approach, which is regarded as a natural RCT, can potentially be used to make causal inference [17-22]. MR uses the random assortment of genes during gamete formation as the natural randomisation process. Therefore, genetic variants known to influence $25(\mathrm{OH}) \mathrm{D}$ concentrations can be used to examine causality as they partly determine lifetime exposure to $25(\mathrm{OH}) \mathrm{D}$. Genome-wide association studies (GWAS) have identified several single nucleotide polymorphisms (SNPs) that are associated with plasma $25(\mathrm{OH}) \mathrm{D}$ concentration in relation to genes involved in the synthesis of 25(OH)D (DHCR7 and CYP2R1), transport of $25(\mathrm{OH}) \mathrm{D}$ via vitamin $\mathrm{D}$-binding protein $(\mathrm{GC} /$ $D B P)$, and metabolism of 25(OH)D (CYP24A1) [23, 24].

The aims of the present study were to test whether higher $25(\mathrm{OH}) \mathrm{D}$ concentrations are observationally associated with cause-specific vascular diseases, or mortality and lipids, and whether such associations are causal among European and Chinese adults.

\section{Methods}

\section{Study populations}

The China Kadoorie Biobank (CKB) is a prospective study of 512,891 individuals, aged 30-79 years, who were enrolled from 5 urban and 5 rural regions in China during 2004-2008. Previous studies have reported the details of the study design, participant characteristics, and survey methods [21]. Information on demographic data, lifestyle, use of medications, and medical history were collected by trained health workers by using laptop-based questionnaires. Blood pressure, weight, and height were measured using standard protocols. Body mass index (BMI) was calculated as body weight in kilogrammes divided by height in metres squared. A $10-\mathrm{mL}$ nonfasting venous blood sample was collected. All participants from CKB provided written informed consent. Ethics approval was obtained from the Peking University and University of Oxford ethics committees.

The Copenhagen General Population Study (CGPS) is a prospective cohort study among Danish population initiated in 2003 [25]. The Copenhagen City Heart Study (CCHS) was initiated in 1976-1978, and followed up in 1981-1983, 1991-1994, and 2001-2003 [13]. Body weight and height was measured by standard methods. Blood pressure was measured in millimetres of mercury with a sphygmomanometer or an automated digital blood pressure monitor (Kivex). Leisure time physical activity was considered in four categories. Smoking information was classified as pack-years smoked. Alcohol consumption was based on average weekly consumption of beer, wine, and liquor. Written informed consent was obtained from participants. Ethics approval was obtained from Danish ethical committees.

\section{Biochemical analysis}

In $\mathrm{CKB}$, plasma $25(\mathrm{OH}) \mathrm{D}$ concentrations were measured using a Beckman Access-2 immunoassay assay on stored EDTA plasma samples in 13,565 individuals. The laboratory participated in the international Vitamin D Quality Assessment Scheme (DEQAS) for 25(OH)D and results had a mean (SD) bias of $-11.8 \%$ (7.5) from the target mean value for the present study. Samples of the CCHS were stored at $-20^{\circ} \mathrm{C}(1981-1983)$, and samples from the CGPS were stored at $-80^{\circ} \mathrm{C}$ (2004-2005). Colorimetric assays (Mannheim, Boehringer Mannheim, Germany or Konelab, Finland, Espoo) were used to measure plasma high-density lipoprotein (HDL) cholesterol and total cholesterol concentrations. 25(OH)D concentrations were measured in both serum and plasma using a chemiluminescent immunoassay, CLIA (DiaSorin, Stillwater, MN, USA). The inter- and intra-assay coefficients of variation were $11 \%$ and $10 \%$, respectively.

\section{SNP selection and genotyping}

A total of 95,680 individuals from the CKB were genotyped using a 384-SNP panel (Illumina Golden Gate). Two synthesis SNPs (CYP2R1-rs10741657 and DHCR7rs12785878), one transport SNP (GC/DBP-rs2282679), and one metabolism SNP (CYP24A1-rs6013897) identified 
for plasma $25(\mathrm{OH}) \mathrm{D}$ concentrations in previously GWAS were included in the SNP panel [20, 21]. The genotyping concordance was $>99.9 \%$ for 2063 pairs of sample replicates and genotype success rates were $99.9 \%$ for each SNP. In the CGPS, genetic variants for $25(\mathrm{OH}) \mathrm{D}$ were selected based on a reported GWAS [23, 24]. The selected SNPs either influence synthesis of pre-vitamin D from 7dehydrocholesterol in the skin or activate transformation from vitamin $\mathrm{D}$ to $25(\mathrm{OH}) \mathrm{D}$ in the liver. TaqMan assays were used for genotyping for DHCR7 (rs7944926 and rs11234027) and CYP2R1 (rs10741657 and rs12794714). Sequencing of randomly selected samples verified the genotypes. All genotype call rates were higher than $99 \%$ complete.

\section{Outcomes}

The present study examined major coronary events (fatal ischaemic heart disease [ICD-10: I20-I25] or nonfatal myocardial infarction [I21-I23]), major vascular events [I60 and I64], intracerebral haemorrhage (I61), ischaemic stroke (I63), and cardiovascular death (I00-I99) as the primary outcomes in the CKB. Data on disease outcomes were identified from claims to the national health insurance system and from local chronic disease registries and disease surveillance point system death registries. Trained staff members, blinded to the baseline information, used International Statistical Classification of Diseases and Related Health Problems (ICD-10) to identify the underlying causes of death. The deaths were grouped into several categories: major coronary events [ICD-10: I20-I25], major vascular events [I60 and I64], ischaemic heart disease (I20-I25), myocardial infarction [I21-I23], ischaemic stroke (I63), intracerebral haemorrhage (I61), cancer (C00-C97), diseases of the respiratory system (J00-J99), infections (A00-B99), and all other causes.

In the CGPS and CCHS studies, information on diagnoses of vascular diseases such as myocardial infarction (ICD-8: 410; ICD-10: I21-I22) and ischaemic heart disease (ICD-8: 410 to 414, and ICD-10: I20 to I25) were verified based on information on diagnoses entered in the national Danish Patient Registry and hospital admissions. The national Danish Causes of Death Registry records were used to identify the causes of death as well as contributing causes of death [12]. We used underlying causes of death to classify deaths as due to cardiovascular (ICD-8 390458, ICD-10 I00-I99) and cancer (ICD-8 140-209, ICD-10 C00-C97) [13].

\section{Statistical methods}

For observational analyses, data were available on 13,565 participants with plasma $25(\mathrm{OH}) \mathrm{D}$ levels for observational analyses, and data were available on 82,464 participants with all genotypes. Of these, 3397 had data on both 25(OH)D plasma levels and genotypes in CKB. We included 25,621 participants and 10,271 participants with plasma 25(OH)D levels in CGPS and CCHS, respectively; in genetic analyses, we included 106,911 participants with all genotypes. Of these, 25,332 had both $25(\mathrm{OH}) \mathrm{D}$ plasma levels and genotypes in CGPS. Cox proportional hazard regression models were used to analysing the relationship between quartile groups of 25(OH)D levels and risk of disease. We used Schoenfeld residual test to examine the proportional hazard assumption. All results $(P>0.05)$ showed that the Cox models were acceptable. Multivariable Cox regression analyses included age (years), sex (male or female), smoking status (current smoker), alcohol intake (current drinker), season, region, systolic blood pressure (SBP), physical activity (low activity) in the CKB, and age (years), sex (male or female), season, SBP, physical activity (low activity), smoking status (current smoker), and alcohol intake (current drinker) in the CGPS and CCHS.

Genotype distributions in each study did not differ from the Hardy-Weinberg equilibrium. Linear regression was used to examine the per allele effects of each SNP on plasma 25(OH)D concentrations. Genetic scores were estimated for the two synthesis SNPs and all four SNPs. The selected SNPs are not in linkage disequilibrium (LD). F-statistics were used to estimate the strength of associations of each SNP with 25(OH)D concentrations, and F-statistics $>10$ were considered strong. We also used linear regression to assess the relationship of each SNP with plasma lipids and lipoproteins. All genetic analyses were adjusted for age at baseline, sex, season, and region. Analyses of individual SNPs and genetic scores with outcomes were estimated using Cox proportional hazard regression models. The per allele effects of each SNP on plasma 25(OH)D concentrations were expressed as the differences in $25(\mathrm{OH}) \mathrm{D}$ concentrations per copy of the $25(\mathrm{OH}) \mathrm{D}$ raising allele.

A two-stage least squared regression model using genotype individually and combined allele scores as instruments was used to examine the influence of a $25 \mathrm{nmol} / \mathrm{L}$ increase in $25(\mathrm{OH}) \mathrm{D}$ concentrations on risk of outcomes. We used the DHCR7/CYP2R1 genetic score as the main instrumental variable. We calculated causal estimates of genetically determined hazard ratios (HRs) by using the Wald-type estimator, which calculates the ratio of the outcome allele score log hazard ratios to the exposure allele score coefficient and then exponentiates the ratio to a hazard ratio. For combination of CVD and lipid data from Asians and Europeans, meta-analyses were conducted. We assessed between-study heterogeneity via Cochrane's $Q$ - and $I^{2}$-statistics. A random-effect meta-analysis was used if $I^{2}>0.25$, otherwise, a fixed-effect model was used [26].

Sensitivity analyses were performed for three-SNP and four-SNP genetic scores to examine the potential effects of pleiotropy using the MR-Egger regression method 
[27]. We used SAS version 9.2 and $\mathrm{R}$ version 3.01. We included 20 diseases or cause-specific mortality as primary outcomes. The threshold of significance was $P<0.0025(0.05 / 20)$ after Bonferroni correction. $P \leq 0.05$ but above the threshold of Bonferroni corrected significance was considered as a suggestive causal association.

\section{Results}

\section{Characteristics of the CKB, CGPS, and CCHS populations} Among the 82,464 CKB individuals and 106,911 CGPS individuals in the genetic study, the mean (SD) of age was 51.4 (10.6) and 58.0 (13.1) years, $61 \%$ and $55 \%$ were women, and the mean (SD) of BMI was 23.7 (3.4) and 26.1 (4.3) $\mathrm{kg} / \mathrm{m}^{2}$, respectively. The overall mean (SD) plasma 25(OH)D concentrations were $63.7(26.6) \mathrm{nmol} / \mathrm{L}, 55.3$ (26.0) nmol/L, 44.3 (24.1) $\mathrm{nmol} / \mathrm{L}$ in CKB, CGPS, and CCHS, respectively (Table 1).

\section{Observational associations}

In $\mathrm{CKB}$, plasma $25(\mathrm{OH}) \mathrm{D}$ levels were not statistically associated with major vascular events (multivariable adjusted HR per $25 \mathrm{nmol} / \mathrm{L}$ higher plasma 25(OH)D, 1.02
[95\% CI, 0.98,1.07]), major coronary events (HR, 0.98 [95\% CI, 0.89,1.07]), myocardial infarction (HR, 0.99 [95\% CI, 0.89,1.12]), stroke (HR, 1.04 [95\% CI, 0.99,1.10]), ischaemic stroke (HR, 0.99[95\% CI, 0.93,1.07]), subarachnoid haemorrhage (HR, 1.24 [95\% CI, 0.83,1.85]), and ischaemic heart disease (HR, 0.98 [95\% CI, 0.89,1.07]) after adjustment for age, sex, smoking status, alcohol intake, season, region, SBP, and physical activity. However, each $25 \mathrm{nmol} / \mathrm{L}$ higher plasma $25(\mathrm{OH}) \mathrm{D}$ concentration was associated with about a 9\% higher risk of intracerebral haemorrhage (HR, 1.09 [95\% CI, 1.01,1.18]) (Table 2). Likewise, plasma 25(OH)D levels were not significantly associated with cancer, respiratory diseases, infection-cause mortality, or all-cause mortality after adjustment for confounding factors (Table 3). In addition, each $25 \mathrm{nmol} /$ $\mathrm{L}$ higher plasma $25(\mathrm{OH}) \mathrm{D}$ concentration was associated with a $1.52 \mathrm{mg} / \mathrm{dL}$ higher apoA, $0.04 \mathrm{mg} / \mathrm{dL}$ higher apoB, and $0.03 \mathrm{mmol} / \mathrm{L}(1 \mathrm{mg} / \mathrm{dL})$ higher HDL cholesterol, but a $0.05 \mathrm{mmol} / \mathrm{L}(2 \mathrm{mg} / \mathrm{dL})$ lower total cholesterol, 0.03 $\mathrm{mmol} / \mathrm{L}(1 \mathrm{mg} / \mathrm{dL})$ lower LDL cholesterol, and a 0.16 $\mathrm{mmol} / \mathrm{L}(14 \mathrm{mg} / \mathrm{dL})$ lower triglycerides (Table 4$)$.

In CGPS and CCHS, each $25 \mathrm{nmol} / \mathrm{L}$ higher plasma $25(\mathrm{OH}) \mathrm{D}$ concentration was associated with about a $10 \%$

Table 1 Characteristics for all individuals with 25(OH)D measured or with genetic data

\begin{tabular}{|c|c|c|c|c|c|}
\hline \multirow{2}{*}{$\begin{array}{l}\text { Baseline } \\
\text { Characteristic }\end{array}$} & \multicolumn{3}{|c|}{ All individuals with $25(\mathrm{OH}) \mathrm{D}$ measured } & \multicolumn{2}{|c|}{ Individuals with genetic data } \\
\hline & $\overline{C K B}$ & CGPS & $\mathrm{CCHS}$ & $\overline{C K B}$ & CGPS \\
\hline Demographic & $(n=13,565)$ & $(n=25,621)$ & $(n=10,271)$ & $(n=82,464)$ & $(n=106,911)$ \\
\hline Age $(S D)$, years & $53.2(11.2)$ & $58.5(13.0)$ & $56.7(11.9)$ & $51.4(10.6)$ & $58.0(13.1)$ \\
\hline Women, \% & 49.2 & 54.9 & 55.7 & 60.5 & 55 \\
\hline Current smoker, \% & 47.2 & 20.5 & 58.4 & 36.9 & 17.1 \\
\hline Current drinker, \% & 56.8 & 88.3 & 69 & 53.7 & 89.1 \\
\hline Low physical activity $^{*}$ & 18.15 & 6.8 & 16.8 & 18.15 & 6.2 \\
\hline BMI (SD), $\mathrm{kg} / \mathrm{m}^{2}$ & $23.6(3.5)$ & $26.1(4.3)$ & $25.3(4.2)$ & $23.7(3.4)$ & $26.1(4.3)$ \\
\hline $\mathrm{SBP}(\mathrm{SD}), \mathrm{mmHg}$ & $140.2(25.9)$ & $141.1(21.1)$ & $140.5(21.6)$ & $131.2(21.3)$ & $141.6(21.4)$ \\
\hline $\mathrm{DBP}(\mathrm{SD}), \mathrm{mmHg}$ & $82.2(14.5)$ & $83.9(11.4)$ & $85.1(12.1)$ & $77.8(11.2)$ & $84.3(11.5)$ \\
\hline \multicolumn{6}{|l|}{ Doctor diagnosed prior disease, $\%$} \\
\hline Heart disease & 0 & 6.1 & 3.5 & 3 & 5.8 \\
\hline Stroke & 0 & 1.4 & 1 & 1.8 & 1.3 \\
\hline Hypertension & 15.9 & 60.9 & 56.1 & 11.5 & 60.3 \\
\hline Diabetes & 3.7 & 4.5 & 3.3 & 3.2 & 4.2 \\
\hline Cancer & 0 & 6.5 & 4 & 0.5 & 6.9 \\
\hline \multicolumn{6}{|l|}{ Current medication, $\%$} \\
\hline Statin use & 0 & 11.6 & NA & 0.2 & 12.1 \\
\hline Aspirin use & 1.3 & 12.7 & NA & 1.1 & 11.7 \\
\hline Blood pressure lowering & 6.1 & 20.3 & 10.8 & 4.8 & 19.8 \\
\hline Plasma 25(OH)D (SD), nmol/L & 83.7 (26.6) & $55.3(26.0)$ & $44.3(24.1)$ & $83.4(26.2)$ & $55.3(26.1)$ \\
\hline
\end{tabular}

CKB the China Kadoorie Biobank, CGPS the Copenhagen General Population Study, CCHS the Copenhagen City Heart Study, SD standard deviation, BMI body mass index, SBP systolic blood pressure, DBP diastolic blood pressure

*Self-reported passivity or less than $2 \mathrm{~h}$ of light physical activity a week in CGPS and CCHS; MET-h/day < 8.4 (the bottom quintile) in CKB

A total of 3397 individuals in the genetic study in CKB had plasma 25(OH)D concentrations measured

25(OH)D: $1 \mathrm{ng} / \mathrm{ml}=2.496 \mathrm{nmol} / \mathrm{L}$ 
Table 2 Observational association of $25(\mathrm{OH})$ D with risk of vascular diseases in CKB, CGPS, and CCHS

\begin{tabular}{|c|c|c|c|c|c|c|c|}
\hline \multirow[t]{3}{*}{ Diseases } & \multicolumn{2}{|c|}{ Continuous 25(OH)D per $25 \mathrm{nmol} / \mathrm{L}$} & \multicolumn{4}{|c|}{$25(\mathrm{OH}) \mathrm{D}$ quartiles $(\mathrm{nmol} / \mathrm{L})$} & \multirow{3}{*}{$\begin{array}{l}P \text { for } \\
\text { trend }\end{array}$} \\
\hline & \multirow{2}{*}{$\begin{array}{l}\text { No. of all } \\
\text { events }\end{array}$} & \multirow[t]{2}{*}{$\mathrm{HR}(95 \% \mathrm{Cl})$} & Quartile 1 & Quartile 2 & Quartile 3 & Quartile 4 & \\
\hline & & & HR $(95 \% \mathrm{Cl})$ & HR $(95 \% \mathrm{Cl})$ & HR $(95 \%$ Cl) & HR $(95 \%$ Cl) & \\
\hline \multicolumn{8}{|l|}{ CKB } \\
\hline Major vascular event & 3868 & $1.02(0.98,1.07)$ & 1.00 & $1.00(0.89,1.12)$ & $1.08(0.95,1.22)$ & $1.03(0.90,1.18)$ & 0.344 \\
\hline Major coronary event & 1024 & $0.98(0.89,1.07)$ & 1.00 & $0.89(0.71,1.13)$ & $0.99(0.78,1.27)$ & $0.85(0.66,1.12)$ & 0.614 \\
\hline Myocardial infarction & 662 & $0.99(0.89,1.12)$ & 1.00 & $0.94(0.69,1.27)$ & $1.05(0.77,1.43)$ & $0.99(0.71,1.38)$ & 0.925 \\
\hline Stroke & 2982 & $1.04(0.99,1.10)$ & 1.00 & $1.01(0.89,1.15)$ & $1.08(0.94,1.24)$ & $1.08(0.93,1.26)$ & 0.154 \\
\hline Ischaemic stroke & 1776 & $0.99(0.93,1.07)$ & 1.00 & $0.95(0.81,1.11)$ & $1.04(0.88,1.23)$ & $0.96(0.79,1.16)$ & 0.882 \\
\hline Intracerebral haemorrhage & 1308 & $1.09(1.01,1.18)$ & 1.00 & $1.04(0.84,1.29)$ & $1.08(0.86,1.35)$ & $1.19(0.93,1.51)$ & 0.029 \\
\hline Subarachnoid haemorrhage & 56 & $1.24(0.83,1.85)$ & 1.00 & $1.05(0.37,2.97)$ & $0.42(0.10,1.72)$ & $1.38(0.42,4.54)$ & 0.291 \\
\hline Ischaemic heart disease & 1024 & $0.98(0.89,1.07)$ & 1.00 & $0.90(0.71,1.15)$ & $1.04(0.81,1.32)$ & $0.84(0.64,1.10)$ & 0.596 \\
\hline \multicolumn{8}{|l|}{ CGPS and CCHS } \\
\hline Cardiovascular disease & 12,110 & $0.90(0.88,0.93)$ & 1.00 & $0.92(0.86,0.98)$ & $0.85(0.79,0.91)$ & $0.79(0.73,0.86)$ & $1.3 \times 10^{-9}$ \\
\hline Myocardial infarction & 4316 & $0.88(0.84,0.93)$ & 1.00 & $0.86(0.77,0.96)$ & $0.81(0.72,0.91)$ & $0.74(0.65,0.85)$ & $5.8 \times 10^{-10}$ \\
\hline Stroke & 4490 & $0.93(0.90,0.98)$ & 1.00 & $0.97(0.87,1.08)$ & $0.87(0.77,0.98)$ & $0.89(0.78,1.02)$ & 0.022 \\
\hline Ischaemic stroke & 3766 & $0.93(0.88,0.98)$ & 1.00 & $0.98(0.87,1.11)$ & $0.84(0.73,0.96)$ & $0.90(0.78,1.04)$ & 0.028 \\
\hline Intracerebral haemorrhage & 542 & $1.05(0.90,1.19)$ & 1.00 & $0.96(0.69,1.33)$ & $1.14(0.82,1.61)$ & $0.96(0.65,1.41)$ & 0.87 \\
\hline Ischaemic heart disease & 9362 & $0.90(0.86,0.93)$ & 1.00 & $0.91(0.84,0.98)$ & $0.86(0.79,0.93)$ & $0.77(0.70,0.84)$ & $1.4 \times 10^{-11}$ \\
\hline
\end{tabular}

Multivariable cox proportional hazard regression models were used to examine the association between quartile groups of $25(\mathrm{OH}) \mathrm{D}$ concentrations and risk of vascular diseases

All values are adjusted for age (years), sex (male or female), smoking status (current smoker), alcohol intake (current drinker), season, region, SBP, and physical activity (low activity) in CKB

All values are adjusted for age (years), sex (male or female), latitude, season, SBP, physical activity (METs, h/day), smoking status (never smoker, occasional smoker, former smoker, or regular smoker), and alcohol intake (non-drinker, occasional drinker, former drinker, or regular drinker) in CGPS and CCHS

lower risk of CVD (multivariable adjusted HR, 0.90 [95\% $\mathrm{CI}, 0.88,0.93]$ ), $12 \%$ lower risk of myocardial infarction (HR, 0.88 [95\% CI, 0.84,0.93]), 7\% lower risk of stroke (HR, 0.93 [95\% CI, 0.90,0.98]), 7\% lower risk of ischaemic stroke ( $\mathrm{HR}, 0.93$ [95\% CI, 0.88,0.98]), and 10\% lower risk of ischaemic heart disease (HR, 0.90 [95\% CI, 0.86,0.93]), but not with intracerebral haemorrhage (HR, 1.05 [95\% CI, 0.90, 1.19]) (Table 2). Individuals in the top quintile of plasma $25(\mathrm{OH}) \mathrm{D}$ concentrations had a $21 \%$ lower risk of incident CVD (HR, 0.79 [95\% CI, 0.73, 0.86) compared with those in the bottom quintile. For specific types of CVD, the adjusted HR for the top vs bottom quintile of plasma 25(OH)D concentrations were HR 0.74 [95\% CI, 0.65,0.85] for myocardial infarction and HR 0.77 [95\% CI, 0.70,0.84] for ischaemic heart disease (Table 2). In addition, a 25 $\mathrm{nmol} / \mathrm{L}$ higher plasma $25(\mathrm{OH}) \mathrm{D}$ concentration was associated with all-cause and cause-specific mortality. The multivariable adjusted HR was $0.90(0.88,0.93)$ for all-cause mortality, $0.90(0.86,0.93)$ for cardiovascular mortality, 0.86 $(0.78,0.98)$ for mortality from myocardial infarction, 0.88 $(0.82,0.95)$ for mortality from ischaemic heart disease, 0.93 $(0.88,0.98$ for cancer mortality, $0.86(0.80,0.93)$ for respiratory disease mortality, and $0.86(0.82,0.90)$ for other mortality (Table 3 ).
Genetic associations with plasma 25(OH)D and risk factors In CKB, plasma $25(\mathrm{OH}) \mathrm{D}$ concentrations per each increasing allele were $1.49 \mathrm{nmol} / \mathrm{L}(\mathrm{SE} 0.38, \mathrm{~F}$-statistic $=89)$ higher for rs12785878 (DHCR7), 0.34 nmol/L (SE 0.15, Fstatistic $=97)$ higher for rs10741657 $(C Y P 2 R 1), 5.10 \mathrm{nmol} /$ L (SE 0.41, F-statistic = 89) higher for $\mathrm{rs} 2282679(\mathrm{GC} /$ DBP), $0.95 \mathrm{nmol} / \mathrm{L}(\mathrm{SE} 0.27$, F-statistic $=278.53)$ higher for two-SNP score (rs12785878 + rs10741657), and 1.84 $\mathrm{nmol} / \mathrm{L}$ (SE 0.21, F-statistic $=69$ ) higher for four-SNP score (Fig. 1 and Additional file 1: Table S1). In CGPS, genetic variants and genetic score were also significantly associated with plasma 25(OH)D concentrations (Fig. 1 and Additional file 1: Table S1). None of the SNPs was associated with differences in confounders such as age, gender, season, region, smoking, drinking, physical activity, blood pressure, and BMI in CKB or in CGPS (Additional file 1: Tables S2 \& S3).

\section{Causal associations with cause-specific vascular disease or mortality}

In both CKB and CGPS, there was no significant genetic association of the DHCR7/CYP2R1 risk score with cause-specific vascular disease (Additional file 1: Tables S4 and S5) or mortality (Additional file 1: Table S6 and S7) 
Table 3 Observational association of 25(OH)D with risk of mortality in the CKB, CGPS, and CCHS

\begin{tabular}{|c|c|c|c|c|c|c|c|}
\hline \multirow[t]{3}{*}{ Mortality } & \multicolumn{2}{|c|}{ Continuous $25(\mathrm{OH}) \mathrm{D}$ per $25 \mathrm{nmol} / \mathrm{L}$} & \multicolumn{4}{|c|}{$25(\mathrm{OH}) \mathrm{D}$ quartiles $(\mathrm{nmol} / \mathrm{L})$} & \multirow{3}{*}{$\begin{array}{l}P \text { for } \\
\text { trend }\end{array}$} \\
\hline & \multirow{2}{*}{$\begin{array}{l}\text { No. of all } \\
\text { events }\end{array}$} & \multirow[t]{2}{*}{ HR $(95 \% \mathrm{Cl})$} & \multirow{2}{*}{$\begin{array}{l}\text { Quartile } 1 \\
\text { HR (95\% Cl) }\end{array}$} & \multirow{2}{*}{$\begin{array}{l}\text { Quartile } 2 \\
\text { HR (95\% Cl) }\end{array}$} & \multirow{2}{*}{$\begin{array}{l}\text { Quartile } 3 \\
\text { HR (95\% Cl) }\end{array}$} & \multirow{2}{*}{$\begin{array}{l}\text { Quartile } 4 \\
\text { HR (95\% Cl) }\end{array}$} & \\
\hline & & & & & & & \\
\hline \multicolumn{8}{|l|}{ CKB } \\
\hline All-cause mortality & 3868 & $1.02(0.97,1.06)$ & 1.00 & $0.95(0.84,1.07)$ & $0.97(0.85,1.10)$ & $0.95(0.83,1.09)$ & 0.458 \\
\hline \multicolumn{8}{|l|}{ Vascular death outcomes } \\
\hline Major vascular event & 3048 & $1.04(0.99,1.09)$ & 1.00 & $0.93(0.81,1.07)$ & $0.97(0.84,1.12)$ & $1.00(0.86,1.17)$ & 0.158 \\
\hline Major coronary event & 1262 & $1.05(0.97,1.14)$ & 1.00 & $0.95(0.77,1.18)$ & $0.98(0.79,1.23)$ & $1.13(0.89,1.42)$ & 0.186 \\
\hline Myocardial infarction & 898 & $1.04(0.94,1.14)$ & 1.00 & $0.90(0.70,1.16)$ & $1.02(0.79,1.33)$ & $1.02(0.77,1.36)$ & 0.427 \\
\hline Stroke & 1590 & $1.06(0.98,1.13)$ & 1.00 & $0.95(0.78,1.14)$ & $1.01(0.82,1.23)$ & $1.01(0.82,1.26)$ & 0.136 \\
\hline Ischaemic stroke & 118 & $1.06(0.84,1.33)$ & 1.00 & $0.58(0.30,1.12)$ & $0.61(0.30,1.21)$ & $0.84(0.42,1.67)$ & 0.622 \\
\hline Intracerebral haemorrhage & 1416 & $1.05(0.98,1.14)$ & 1.00 & $0.97(0.80,1.19)$ & $1.06(0.85,1.32)$ & $1.04(0.82,1.31)$ & 0.166 \\
\hline Subarachnoid haemorrhage & 68 & $1.01(0.98,1.03)$ & 1.00 & $0.95(0.18,4.93)$ & $1.52(0.32,7.33)$ & $1.10(0.18,6.83)$ & 0.685 \\
\hline Ischaemic heart disease & 1262 & $1.05(0.97,1.14)$ & 1.00 & $0.95(0.77,1.18)$ & $0.98(0.79,1.23)$ & $1.13(0.89,1.42)$ & 0.186 \\
\hline \multicolumn{8}{|l|}{ Non-vascular death outcomes } \\
\hline Cancer & 370 & $1.00(0.99,1.01)$ & 1.00 & $1.22(0.81,1.83)$ & $1.17(0.76,1.79)$ & $1.00(0.63,1.58)$ & 0.818 \\
\hline Respiratory diseases & 46 & $1.00(0.99,1.01)$ & 1.00 & $0.91(0.47,1.73)$ & $1.28(0.68,2.42)$ & $0.75(0.35,1.60)$ & 0.798 \\
\hline Infections & 64 & $1.00(0.98,1.02)$ & 1.00 & $0.51(0.08,3.17)$ & $0.38(0.06,2.53)$ & $1.00(0.21,4.81)$ & 0.946 \\
\hline \multicolumn{8}{|l|}{ CGPS and CCHS } \\
\hline All-cause mortality & 10,845 & $0.90(0.88,0.93)$ & 1.00 & $0.81(0.77,0.85)$ & $0.79(0.75,0.83)$ & $0.77(0.73,0.82)$ & $2 \times 10^{-20}$ \\
\hline \multicolumn{8}{|l|}{ Vascular death outcomes } \\
\hline Cardiovascular disease & 3303 & $0.90(0.86,0.93)$ & 1.00 & $0.85(0.78,0.93)$ & $0.78(0.71,0.87)$ & $0.80(0.72,0.90)$ & $1.9 \times 10^{-8}$ \\
\hline Myocardial infarction & 676 & $0.86(0.78,0.98)$ & 1.00 & $0.68(0.55,0.83)$ & $0.78(0.63,0.97)$ & $0.70(0.54,0.90)$ & 0.0037 \\
\hline Stroke & 743 & $0.93(0.86,1.03)$ & 1.00 & $0.97(0.81,1.17)$ & $0.74(0.60,0.92)$ & $1.00(0.79,1.26)$ & 0.28 \\
\hline Ischaemic stroke & 385 & $0.88(0.78,1.00)$ & 1.00 & $1.06(0.82,1.36)$ & $0.65(0.48,0.89)$ & $0.96(0.69,1.34)$ & 0.18 \\
\hline Intracerebral haemorrhage & 178 & $1.05(0.88,1.25)$ & 1.00 & $1.06(0.71,1.57)$ & $0.96(0.62,1.49)$ & $1.16(0.72,1.86)$ & 0.71 \\
\hline Ischaemic heart disease & 1395 & $0.88(0.82,0.95)$ & 1.00 & $0.80(0.70,0.92)$ & $0.80(0.69,0.93)$ & $0.76(0.63,0.90)$ & $6 \times 10^{-4}$ \\
\hline \multicolumn{8}{|l|}{ Non-vascular death outcomes } \\
\hline Cancer & 3127 & $0.93(0.88,0.98)$ & 1.00 & $0.86(0.78,0.94)$ & $0.87(0.78,0.96)$ & $0.79(0.71,0.89)$ & $9.7 \times 10^{-5}$ \\
\hline Respiratory diseases & 1065 & $0.86(0.80,0.93)$ & 1.00 & $0.69(0.59,0.82)$ & $0.67(0.56,0.80)$ & $0.71(0.59,0.87)$ & $6.6 \times 10^{-4}$ \\
\hline Infections & 430 & $0.84(0.76,0.95)$ & 1.00 & $0.76(0.59,0.98)$ & $0.85(0.66,1.11)$ & $0.73(0.53,1.00)$ & 0.082 \\
\hline All other causes & 2463 & $0.86(0.82,0.90)$ & 1.00 & $0.73(0.66,0.81)$ & $0.71(0.63,0.79)$ & $0.68(0.59,0.78)$ & $4.6 \times 10^{-10}$ \\
\hline
\end{tabular}

Multivariable cox proportional hazard regression models were used to examine the association between quartile groups of 25(OH)D concentrations and risk of mortality

All values are adjusted for age (years), sex (male or female), smoking status (current smoker), alcohol intake (current drinker), season, region, SBP, and physical activity (low activity) in CKB

All values are adjusted for age (years), sex (male or female), latitude, season, SBP, physical activity (METs, h/day), smoking status (never smoker, occasional smoker, former smoker, or regular smoker), and alcohol intake (non-drinker, occasional drinker, former drinker, or regular drinker) in CGPS and CCHS

after Bonferroni correction. Instrumental variable analysis did not show any significant causal association of genetically predicted $25(\mathrm{OH}) \mathrm{D}$ with any cause-specific vascular disease in CKB and CGPS (Fig. 2). Furthermore, genetically predicted $25(\mathrm{OH}) \mathrm{D}$ was not significantly associated with cause-specific mortality in CKB and CGPS (Fig. 3). However, the instrumental variable analysis indicated a marginal association for all-cause mortality per a $25 \mathrm{nmol} /$ L higher in genetically predicted $25(\mathrm{OH}) \mathrm{D}$ concentrations $(0.98[0.96,1.00])$ (Fig. 3). Sensitivity analyses yielded similar findings for genetically instrumented differences in $25(\mathrm{OH}) \mathrm{D}$ concentrations using the individual SNP or fourSNP score for each disease (Additional file 1: Table S4 to S7).

In addition, instrumental variable analyses showed significant associations with total and LDL cholesterol in CKB (Fig. 4 and Additional file 1: Table S8). Each 25 $\mathrm{nmol} / \mathrm{l}$ genetic increase in $25(\mathrm{OH}) \mathrm{D}$ concentrations was associated with $0.058 \mathrm{mmol} / \mathrm{L}(0.22 \mathrm{mg} / \mathrm{dL})$ and 0.034 $\mathrm{mmol} / \mathrm{L}(0.13 \mathrm{mg} / \mathrm{dL})$ lower total and LDL cholesterol in 
Table 4 Observational association of 25(OH)D with lipids in CKB, CGPS, and CCHS

\begin{tabular}{|c|c|c|c|c|c|c|}
\hline \multirow[t]{3}{*}{ Lipids } & \multirow{3}{*}{$\begin{array}{l}\text { Continuous } 25(\mathrm{OH}) \mathrm{D} \text { per } 25 \mathrm{nmol} / \mathrm{L} \\
\text { Beta } \pm \text { SE }\end{array}$} & \multicolumn{4}{|c|}{$25(\mathrm{OH}) \mathrm{D}$ quartiles $(\mathrm{nmol} / \mathrm{L})$} & \multirow{3}{*}{$\begin{array}{l}P \text { for } \\
\text { trend }\end{array}$} \\
\hline & & \multirow{2}{*}{$\begin{array}{l}\text { Quartile } 1 \\
\text { Mean } \pm \text { SD }\end{array}$} & \multirow{2}{*}{$\begin{array}{l}\text { Quartile } 2 \\
\text { Mean } \pm \text { SD }\end{array}$} & \multirow{2}{*}{$\begin{array}{l}\text { Quartile } 3 \\
\text { Mean } \pm \text { SD }\end{array}$} & \multirow{2}{*}{$\begin{array}{l}\text { Quartile } 4 \\
\text { Mean } \pm \text { SD }\end{array}$} & \\
\hline & & & & & & \\
\hline \multicolumn{7}{|l|}{ CKB $(n=17,755)$} \\
\hline Apoa, mg/dL & $1.52 \pm 0.21$ & $127.90 \pm 0.43$ & $129.48 \pm 0.42$ & $129.79 \pm 0.41$ & $130.73 \pm 0.45$ & $<0.0001$ \\
\hline Apob, mg/dL & $0.04 \pm 0.00$ & $84.52 \pm 0.46$ & $85.95 \pm 0.45$ & $84.14 \pm 0.44$ & $81.42 \pm 0.44$ & $<0.0001$ \\
\hline Lpa, mmol/L & $0.01 \pm 0.49$ & $37.94 \pm 1.00$ & $36.40 \pm 0.99$ & $35.17 \pm 0.96$ & $37.15 \pm 1.02$ & 0.984 \\
\hline $\mathrm{TC}, \mathrm{mmol} / \mathrm{L}$ & $-0.05 \pm 0.01$ & $4.65 \pm 0.02$ & $4.74 \pm 0.02$ & $4.66 \pm 0.02$ & $4.53 \pm 0.02$ & $<0.0001$ \\
\hline $\mathrm{HDL}, \mathrm{mmol} / \mathrm{L}$ & $0.03 \pm 0.01$ & $1.20 \pm 0.01$ & $1.22 \pm 0.01$ & $1.23 \pm 0.01$ & $1.26 \pm 0.01$ & $<0.0001$ \\
\hline $\mathrm{LDL}, \mathrm{mmol} / \mathrm{L}$ & $-0.03 \pm 0.01$ & $2.32 \pm 0.01$ & $2.38 \pm 0.01$ & $2.34 \pm 0.01$ & $2.28 \pm 0.02$ & $<0.0001$ \\
\hline $\mathrm{TG}, \mathrm{mmol} / \mathrm{L}$ & $-0.16 \pm 0.02$ & $2.18 \pm 0.04$ & $2.09 \pm 0.03$ & $2.03 \pm 0.03$ & $1.75 \pm 0.03$ & $<0.0001$ \\
\hline \multicolumn{7}{|c|}{ CGPS and CCHS $(n=106,911)$} \\
\hline Apoa, mg/dL* & $0.01 \pm 0.002$ & $160.63 \pm 31.08$ & $161.78 \pm 29.96$ & $163.35 \pm 30.09$ & $164.39 \pm 32.33$ & $<0.0001$ \\
\hline Apob, mg/dL* & $-0.08 \pm 0.002$ & $125.27 \pm 40.06$ & $118.58 \pm 35.59$ & $112.93 \pm 33.17$ & $104.34 \pm 29.31$ & $<0.0001$ \\
\hline Lpa, mg/dL* & $0.32 \pm 0.231$ & $22.99 \pm 31.53$ & $22.80 \pm 30.25$ & $22.83 \pm 32.66$ & $23.90 \pm 31.30$ & 0.15 \\
\hline $\mathrm{TC}, \mathrm{mmol} / \mathrm{L}$ & $-0.14 \pm 0.006$ & $5.85 \pm 1.16$ & $5.80 \pm 1.12$ & $5.71 \pm 1.10$ & $5.53 \pm 1.09$ & $<0.0001$ \\
\hline $\mathrm{HDL}, \mathrm{mmol} / \mathrm{L}$ & $0.06 \pm 0.003$ & $1.38 \pm 0.50$ & $1.46 \pm 0.51$ & $1.51 \pm 0.51$ & $1.56 \pm 0.52$ & $<0.0001$ \\
\hline $\mathrm{LDL}, \mathrm{mmol} / \mathrm{L}^{*}$ & $-0.15 \pm 0.006$ & $3.44 \pm 1.00$ & $3.36 \pm 0.96$ & $3.27 \pm 0.95$ & $3.09 \pm 0.91$ & $<0.0001$ \\
\hline $\mathrm{TG}, \mathrm{mmol} / \mathrm{L}^{*}$ & $-0.26 \pm 0.007$ & $2.08 \pm 1.49$ & $1.78 \pm 1.10$ & $1.60 \pm 0.95$ & $1.39 \pm 0.77$ & $<0.0001$ \\
\hline
\end{tabular}

Linear regression was also used to assess the associations of $25(\mathrm{OH}) \mathrm{D}$ with lipids

All values are adjusted for age (years), sex (male or female), smoking status (current smoker), alcohol intake (current drinker), season, SBP, and physical activity (low activity) in CKB

All values are adjusted for age (years), sex (male or female), latitude, season, region, SBP, physical activity (METs, h/day), smoking status (never smoker, occasional smoker, former smoker, or regular smoker), and alcohol intake (non-drinker, occasional drinker, former drinker, or regular drinker) in CGPS and CCHS

*Data only available in CGPS

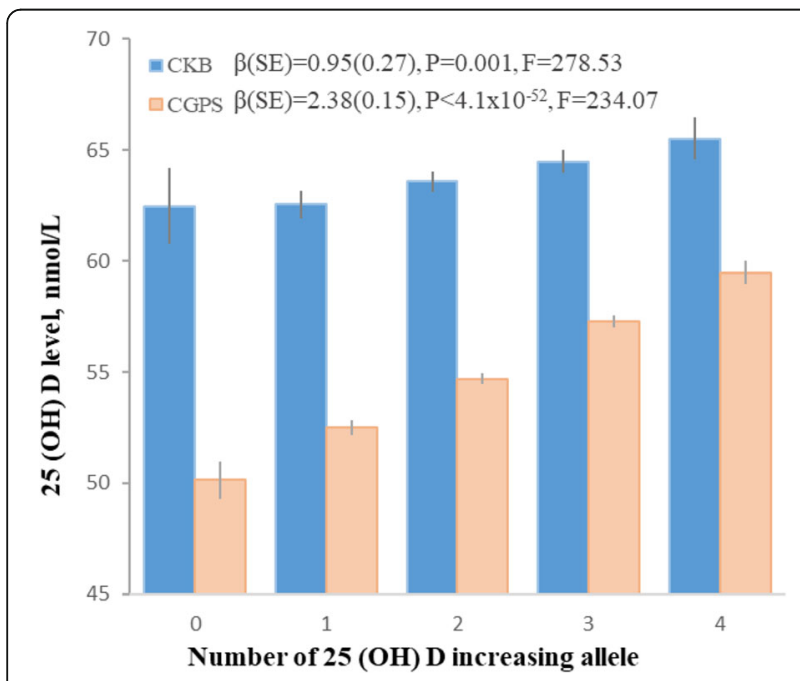

Fig. 1 Genetic association with 25(OH)D (nmol/L) in CKB and CGPS. Data are presented as beta \pm SE. Linear regression was used to assess the per allele effect of each SNP or genetic score on plasma 25(OH)D concentrations. All values are adjusted for age, sex, and season and stratified by region. CKB, the China Kadoorie Biobank; CGPS, the Copenhagen General Population Study
CKB. However, we did not observe causal associations for lipids and lipoproteins in CGPS and pooled cohorts (Fig. 4 and Additional file 1: Table S9).

\section{Discussion}

The novel findings of this MR study involving 99,012 Chinese adults and 106,911 European adults suggest that genetically higher plasma $25(\mathrm{OH}) \mathrm{D}$ levels were not associated with lower risk of cause-specific vascular disease such as myocardial infarction, ischaemic heart disease, ischaemic stroke, stroke, intracerebral haemorrhage, and subarachnoid haemorrhage among both Chinese and European adults. Likewise, 25(OH)D may not influence lipid levels. Our results suggest that the inverse associations of vitamin D with cause-specific vascular disease and lipids could be the results of confounding.

Using an MR approach free from confounding and reverse causation, our study for the first time showed that genetically predicted $25(\mathrm{OH}) \mathrm{D}$ was not associated with intracerebral haemorrhage, subarachnoid haemorrhage, stroke, and ischaemic stroke, thus suggesting that vitamin D may not play a causal role in development of cause-specific vascular disease in Chinese or Europeans. The results suggest that individuals should be cautious about long-term supplementation with high doses of 


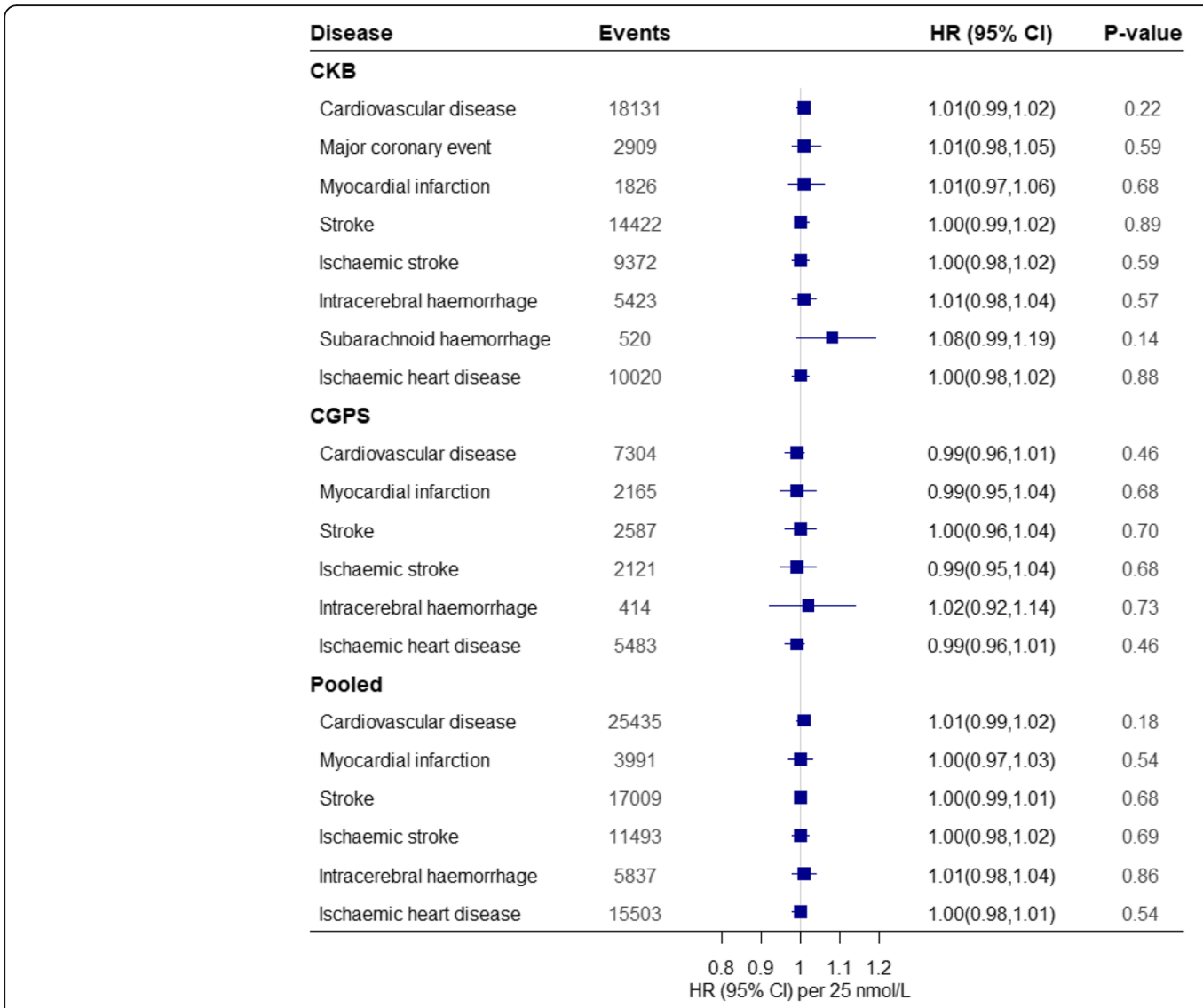

Fig. 2 Instrumental variable estimates for vascular diseases. Analyses of two-SNP score with vascular diseases were estimated using cox proportional hazard regression models. We used a two-SNP score as instrument to estimate the influence of a $25 \mathrm{nmol} / \mathrm{L}$ increase in $25(\mathrm{OH}) \mathrm{D}$ concentrations on risk of vascular diseases. We calculated instrumental variable estimates of genetically determined hazard ratios by using the Wald-type estimator, which involves taking the ratio of the gene-outcome log hazard ratios to the gene-exposure coefficient and then exponentiating to express it as a hazard ratio. Two-SNP score was calculated based on DHCR7 + CYP2R1: rs 12785878 + rs10741657 in CKB and DHCR7 + CYP2R1: rs7944926 + rs 10741657 in CGPS. The $r^{2}$ between rs12785878 and rs7944926 is 0.87 . All values are adjusted for age, sex, and season and stratified by region

vitamin D3 [10]. The results are consistent with recent MR studies which did not show a causal effect of circulating vitamin $\mathrm{D}$ levels on risk of ischaemic heart disease or myocardial infarction [12], or cardiovascular mortality [13]. Findings from RCTs that do not show a protective effect of vitamin D3 supplementation on cardiovascular outcomes [9-11], supported our findings. However, previous RCTs did not specifically focus on vitamin Ddeficient population. Thus, available evidence is not enough to support cardiovascular benefits or hazards of the commonly used vitamin D doses, but whether higher doses of vitamin D are beneficial is uncertain, or whether vitamin D supplementation has vascular effects in individuals with vitamin $\mathrm{D}$ deficiency remains unclear [9]. It worth noting that there are two major forms of vitamin D: vitamin D2 (ergocalciferol) and vitamin D3 (cholecalciferol). A meta-analysis demonstrated that vitamin D3 is more effective than vitamin D2 in maintaining circulating concentrations of 25(OH)D [28]. Previous randomised double-blind placebo-controlled trial examining the effects of vitamin D2 or D3 supplementation on cardiometabolic risk showed that both vitamin D2 or D3 did not influence blood pressure and cardiovascular risk, but decreased $A p o B$ concentration and pulse wave velocity. Only vitamin D2 decreased total cholesterol [29]. However, so far, no RCT has investigated the benefits of supplemental vitamin D2 or D3 in preventing vascular diseases in Chinese adults. Whether vitamin D2 or D3 supplementation will be beneficial in patients with CVD or high-risk older patients is unknown and warrants further investigation.

Our findings could be explained by previous evidence that low $25(\mathrm{OH}) \mathrm{D}$ concentration is a marker of an unhealthy lifestyle or poor health [30], which has been associated with increased risk of vascular disease. Furthermore, previous MR studies suggest that cardiovascular factors [31], such as high remnant cholesterol, LDL cholesterol [32], and obesity [33], decrease plasma concentrations of $25(\mathrm{OH}) \mathrm{D}$ and increase vascular risk. That means low 


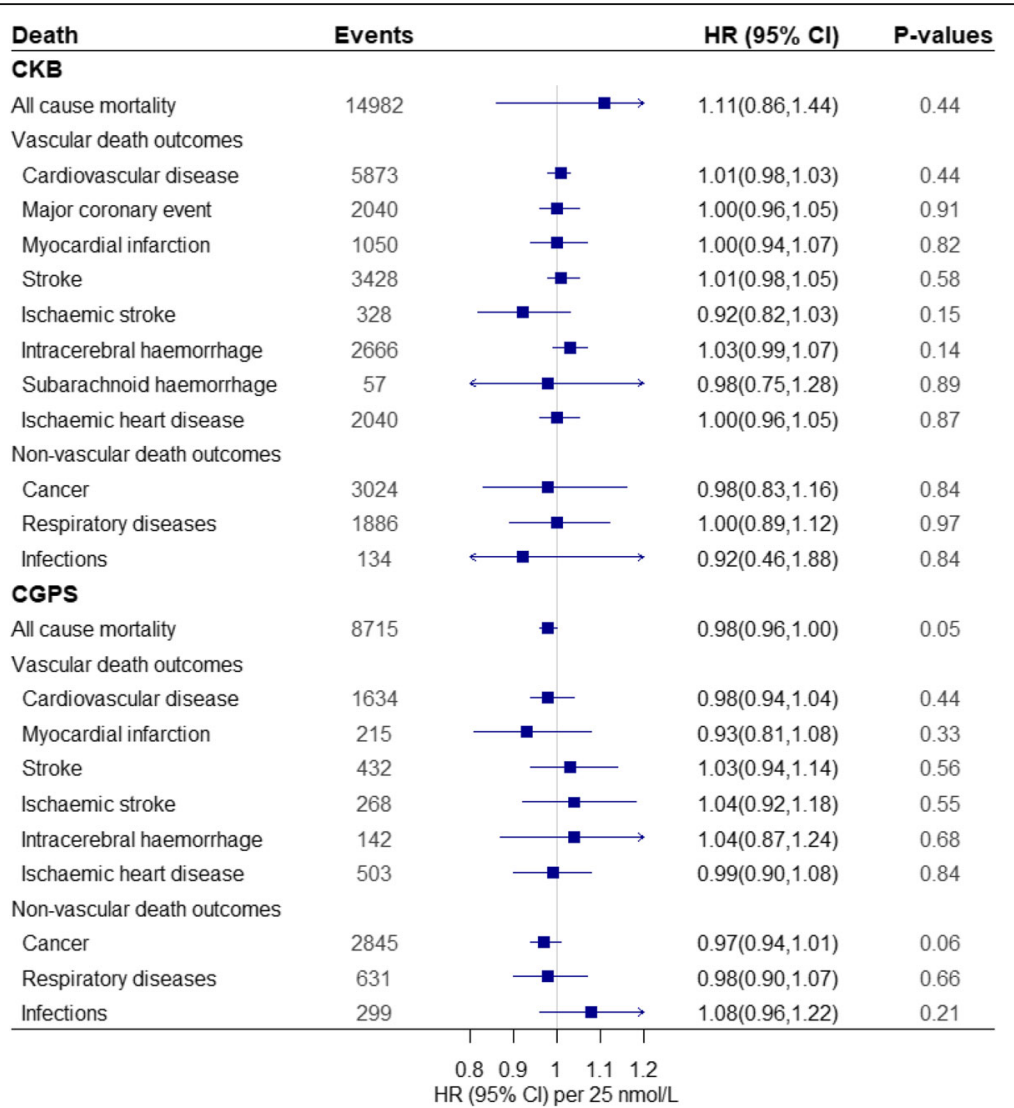

Fig. 3 Instrumental variable estimates for mortality. Analyses of two-SNP score with mortality were estimated using cox proportional hazard regression models. We used a two-SNP score as instrument to estimate the influence of a $25 \mathrm{nmol} / \mathrm{L}$ increase in $25(\mathrm{OH}) \mathrm{D}$ concentrations on risk of mortality. We calculated instrumental variable estimates of genetically determined hazard ratios by using the Wald-type estimator, which involves taking the ratio of the gene-outcome log hazard ratios to the gene-exposure coefficient and then exponentiating to express it as a hazard ratio. Number of individuals in CKB and CGPS are 99,012 and 106,911, respectively. Two-SNP score was calculated based on DHCR7+ CYP2R1: rs $12785878+$ rs 10741657 in CKB and DHCR7 + CYP2R1: rs7944926 + rs10741657 in CGPS. The $r^{2}$ between rs 12785878 and rs7944926 is 0.87. All values are adjusted for age, sex, and season and stratified by region

plasma $25(\mathrm{OH}) \mathrm{D}$ is a consequence of CVD rather than a causal factor. This could explain why we did not observe a causal association in MR analyses, despite strong observational associations in Europeans [4, 5]. In addition, a previous study showed that elevated parathyroid hormone levels identify a population with higher cardiovascular risk. Parathyroid hormone correlates to cardiovascular risk, but not vitamin D [34]. The potential mechanism is that parathyroid hormone induces oxidative stress and affects endothelial function [35]. Therefore, we have to acknowledge that the vitamin D-dependent parathyroid hormone, rather than vitamin $\mathrm{D}$ itself, might be a better predictor of cardiovascular risk. However, we have to acknowledge that the possibility of a non-linear association of vitamin $\mathrm{D}$ with cardiovascular outcomes cannot be excluded as it was not tested in the present MR study.

Although our MR analysis did not show causal effects of $25(\mathrm{OH}) \mathrm{D}$ on all-cause and cause-specific mortality in Chinese, results from Europeans demonstrated that $25(\mathrm{OH}) \mathrm{D}$ might be causally related to all-cause mortality and cancer mortality. Our findings are consistent with a European MR study, which showed that genetically reduced vitamin $\mathrm{D}$ concentrations were associated with increased all-cause, cancer mortality, but not with cardiovascular mortality [13]. Our results in Europeans are also supported by a meta-analysis of 56 RCTs mainly conducted in Europeans, which showed that vitamin D decreased allcause and cancer mortality [11]. However, when different forms of vitamin D were assessed, only vitamin D3 (cholecalciferol) decreased mortality. Vitamin D2 (ergocalciferol) may even increase mortality [11]. It should be noted that recent European MR studies found evidence that genetically determined vitamin $\mathrm{D}$ concentrations were inversely associated with type 2 diabetes [36], and blood pressure and risk of hypertension [37]. These results could at least partly explain the reduced mortality among Europeans observed by us. However, so far, neither RCTs nor MR studies have investigated the causal effect of vitamin D on all-cause and cause-specific mortality in Chinese adults. Our present MR analysis for the first time documented 


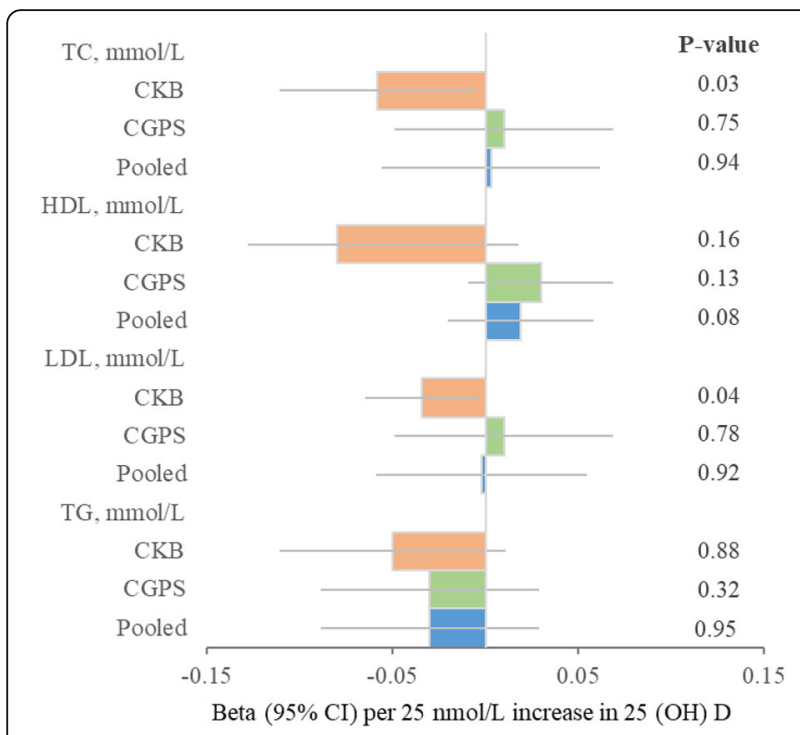

Fig. 4 Instrumental variable estimates for lipids. Linear regression was also used to assess the associations of a two-SNP score with lipids. We used a two-SNP score as instrument to estimate the influence of a 25 $\mathrm{nmol} / \mathrm{L}$ increase in $25(\mathrm{OH}) \mathrm{D}$ concentrations on lipids. We calculated instrumental variable estimates of genetically determined hazard ratios by using the Wald-type estimator, which involves taking the ratio of the gene-outcome coefficient to the gene-exposure coefficient. TwoSNP score was calculated based on DHCR7 + CYP2R1: rs12785878 + rs 10741657 in CKB and DHCR7 + CYP2R1: rs7944926 + rs10741657 in CGPS. The $r^{2}$ between rs 12785878 and rs 7944926 is 0.87 . All values are adjusted for age, sex, and season and stratified by region

that genetically elevated $25(\mathrm{OH}) \mathrm{D}$ was not associated with cause-specific vascular disease or mortality. These findings suggest possible ethnic differences in relationships between $25(\mathrm{OH}) \mathrm{D}$ levels and cancer mortality. A previous study showed that the effect of vitamin D3 supplementation on advanced colorectal adenomas varied according to vitamin $\mathrm{D}$ receptor genotypes [38]. Among the rs7968585AA genotype individuals (26\%), supplemental vitamin D3 reduced risk by $64 \%$, but increased risk by $41 \%$ among individuals with rs7968585 G alleles (74\%) [38]. Therefore, whether benefits from vitamin $\mathrm{D}$ supplementation for the prevention of cancer vary according to vitamin $\mathrm{D}$ receptor genotype is unknown and warrants further investigation.

Previous RCTs have shown inconsistent results for the effects of vitamin D on plasma lipid levels [39-44]. However, it is worth noting that none of the RCTs were specifically conducted for evaluating the effect of supplemental vitamin $\mathrm{D}$ on lipids and lipoproteins. Interestingly, the largest trial (The WHI-Calcium+Vitamin D trial) conducted in 36,282 women showed that patients receiving combined vitamin D and omega-3 fatty acid supplementation had decreased serum very low-density lipoprotein cholesterol and serum triglycerides [42] while $400 \mathrm{IU}$ vitamin D3 plus $1000 \mathrm{mg}$ elemental calcium carbonate daily showed a decrease in LDL cholesterol after 6 weeks of intervention [43]. Consistently, our MR analysis in Chinese adults for the first time showed that each 25 $\mathrm{nmol} / \mathrm{l}$ genetic increase in $25(\mathrm{OH}) \mathrm{D}$ concentration was associated with $0.058 \mathrm{mmol} / \mathrm{L}(0.22 \mathrm{mg} / \mathrm{dL})$ and $0.034 \mathrm{mmol} /$ $\mathrm{L}(0.13 \mathrm{mg} / \mathrm{dL})$ lower total and LDL cholesterol, thus suggesting that $25(\mathrm{OH}) \mathrm{D}$ may play a causal role in lipid metabolism at least in Chinese adults. However, the potential mechanism warrants further investigation.

In the present MR analysis, we considered three assumptions to ensure the validity of our analyses [17, 18, 27]. First, our data demonstrated that the individual SNP and genetic score were associated with 25(OH)D (F statistic > 15), suggesting strong instrumental variables. Second, the instruments should be independent of measured and unmeasured confounders. Indeed, we found no association of genetic variants or score with measured confounders, thus satisfying an important condition for a valid MR experiment. Third, the genetic score should have no pleiotropic effect on the outcomes, which is an important consideration in MR studies. The third assumption may be violated by pleiotropy [45]. The SNPs encoding enzymes involved in the synthesis of $25(\mathrm{OH}) \mathrm{D}$ are believed to be more specific for $25(\mathrm{OH}) \mathrm{D}$, but SNPs involved in the transport of $25(\mathrm{OH}) \mathrm{D}$ may have pleiotropic effects in addition to their effects on plasma $25(\mathrm{OH}) \mathrm{D}$ concentrations alone [31, $46,47]$. However, when we excluded SNPs involved in the transport of $25(\mathrm{OH}) \mathrm{D}$, the association remained unchanged.

Several strengths of the present study merit consideration. This is the first MR analysis to examine the causal association of $25(\mathrm{OH}) \mathrm{D}$ with intracerebral haemorrhage and subarachnoid haemorrhage. This large MR study had a large number of disease events and high-quality $25(\mathrm{OH}) \mathrm{D}$ measurements by well-trained technicians and an appropriate design allowed us to gain sufficient power for estimation of causal effects. Another strength of our study was detailed information on known confounding factors, which allowed us to examine the three MR assumptions. Finally, we used standardised analytic methods to compare the differences in causal effects between Chinese and Europeans.

\section{Conclusion}

In summary, this large-scale analysis provides reliable evidence that higher $25(\mathrm{OH}) \mathrm{D}$ may not play a causal role in developing cause-specific vascular disease in Chinese or Europeans. MR analysis further demonstrated that high vitamin D may not influence lipids.

\section{Additional file}

Additional file 1: Supplemental tables providing additional information on genetic association with 25(OH)D, lipids and CVD. (DOCX $52 \mathrm{~kb}$ ) 


\section{Abbreviations}

CCHS: Copenhagen City Heart Study; CGPS: Copenhagen General Population Study; CKB: China Kadoorie Biobank; GWAS: Genome-wide association studies; MR: Mendelian randomisation; RCT: Randomised intervention trial; SNP: Single nucleotide polymorphisms

\section{Acknowledgements}

The most important acknowledgment is to the participants in the study and the members of the survey teams in each of the 10 regional centres, as well as to the project development and management teams based at Beijing, Oxford, and the 10 regional centres.

Members of the China Kadoorie Biobank collaborative group:

International Steering Committee: Junshi Chen, Zhengming Chen (PI), Robert Clarke, Rory Collins, Yu Guo, Liming Li (PI), Jun Lv, Richard Peto, Robin Walters. International Co-ordinating Centre, Oxford: Daniel Avery, Ruth Boxall, Derrick Bennett, Yumei Chang, Yiping Chen, Zhengming Chen, Robert Clarke, Huaidong Du, Simon Gilbert, Alex Hacker, Mike Hill, Michael Holmes, Andri Iona, Christiana Kartsonaki, Rene Kerosi, Ling Kong, Om Kurmi, Garry Lancaster, Sarah Lewington, Kuang Lin, John McDonnell, Iona Millwood, Qunhua Nie, Jayakrishnan Radhakrishnan, Paul Ryder, Sam Sansome, Dan Schmidt, Paul Sherliker, Rajani Sohoni, Becky Stevens, lain Turnbull, Robin Walters, Jenny Wang, Lin Wang, Neil Wright, Ling Yang, Xiaoming Yang. National Co-ordinating Centre, Beijing: Zheng Bian, Yu Guo, Xiao Han, Can Hou, Jun Lv, Pei Pei, Chao Liu, Yunlong Tan, Canqing Yu. 10 Regional Co-ordinating Centres: Qingdao CDC: Zengchang Pang, Ruqin Gao, Shanpeng Li, Shaojie Wang, Yongmei Liu, Ranran Du, Yajing Zang, Liang Cheng, Xiaocao Tian, Hua Zhang, Yaoming Zhai, Feng Ning, Xiaohui Sun, Feifei Li. Licang CDC: Silu LV, Junzheng Wang, Wei Hou. Heilongjiang Provincial CDC: Mingyuan Zeng, Ge Jiang, Xue Zhou. Nangang CDC: Liqiu Yang, Hui He, Bo Yu, Yanjie Li, Qinai Xu, Quan Kang, Ziyan Guo. Hainan Provincial CDC: Dan Wang, Ximin Hu, Jinyan Chen, Yan Fu, Zhenwang Fu, Xiaohuan Wang. Meilan CDC: Min Weng, Zhendong Guo, Shukuan Wu, Yilei Li, Huimei Li, Zhifang Fu. Jiangsu Provincial CDC: Ming Wu, Yonglin Zhou, Jinyi Zhou, Ran Tao, Jie Yang, Jian Su. Suzhou CDC: Fang liu, Jun Zhang, Yihe Hu, Yan Lu, Liangcai Ma, Aiyu Tang, Shuo Zhang, Jianrong Jin, Jingchao Liu. Guangxi Provincial CDC: Zhenzhu Tang, Naying Chen, Ying Huang. Liuzhou CDC: Mingqiang Li, Jinhuai Meng, Rong Pan, Qilian Jiang, Jian Lan,Yun Liu, Liuping Wei, Liyuan Zhou, Ningyu Chen Ping Wang, Fanwen Meng, Yulu Qin, Sisi Wang. Sichuan Provincial CDC: Xianping Wu, Ningmei Zhang, Xiaofang Chen,Weiwei Zhou. Pengzhou CDC: Guojin Luo, Jianguo Li, Xiaofang Chen, Xunfu Zhong, Jiaqiu Liu, Qiang Sun. Gansu Provincial CDC: Pengfei Ge, Xiaolan Ren, Caixia Dong. Maiji CDC: Hui Zhang, Enke Mao, Xiaoping Wang, Tao Wang, Xi zhang. Henan Provincial CDC: Ding Zhang, Gang Zhou, Shixian Feng, Liang Chang, Lei Fan. Huixian CDC: Yulian Gao, Tianyou He, Huarong Sun, Pan He, Chen Hu, Xukui Zhang, Huifang Wu, Pan He. Zhejiang Provincial CDC: Min Yu, Ruying Hu, Hao Wang. Tongxiang CDC: Yijian Qian, Chunmei Wang, Kaixu Xie, Lingli Chen, Yidan Zhang, Dongxia Pan, Qijun Gu. Hunan Provincial CDC: Yuelong Huang, Biyun Chen, Li Yin, Huilin Liu, Zhongxi Fu, Qiaohua Xu. Liuyang CDC: Xin Xu, Hao Zhang, Huajun Long, Xianzhi Li, Libo Zhang, Zhe Qiu.

\section{Authors' contributions}

All authors read and approved the final manuscript. All authors agree to publish this work.

\section{Funding}

This work was supported by the High-performance Computing Platform of Peking University and grants (2016YFC0900500, 2016YFC0900501, 2016YFC0900504) from the National Key R\&D Program of China. The CKB baseline survey and the first re-survey were supported by a grant from the Kadoorie Charitable Foundation in Hong Kong. The long-term follow-up is supported by grants from the UK Wellcome Trust (202922/Z/16/Z, 088158/Z/ 09/Z, 104085/Z/14/Z), National Natural Science Foundation of China (81390540, 81390544, 81390541), and Chinese Ministry of Science and Technology (2011BAl09B01). The funders had no role in study design, data collection and analysis, decision to publish, or preparation of the manuscript.

\section{Availability of data and materials}

All data generated or analysed during this study are included in this published article and its supplementary information files. Individual participant data are available at www.ckbiobank.org for researchers who meet the criteria for access to released data.

\section{Ethics approval and consent to participate}

All individuals from the CKB provided written informed consent. Ethics approval was obtained from the Peking University and University of Oxford ethics committees. All participants from the Copenhagen City Heart Study, and the Copenhagen General Population Study provided written informed consent and Ethics approval was obtained from Danish ethical committees.

\section{Competing interests}

The authors declare that they have no competing interests.

\section{Author details}

'Department of Epidemiology and Biostatistics, School of Public Health, Peking University Health Science Center, 38 Xueyuan Road, Beijing 100191, China. ${ }^{2}$ Key Laboratory of Molecular Cardiovascular Sciences (Peking University), Ministry of Education, Beijing, China. ${ }^{3}$ Department of Clinical Biochemistry and Copenhagen General Population Study, Copenhagen University Hospital, Herlev and Gentofte Hospital Copenhagen, Copenhagen, Denmark. ${ }^{4}$ Chinese Academy of Medical Sciences, Beijing, China. ${ }^{5}$ Clinical Trial Service Unit \& Epidemiological Studies Unit (CTSU), Nuffield Department of Population Health, University of Oxford, Oxford, UK. ${ }^{6}$ NCDs Prevention and Control Department, Liuzhou Center for Disease Control and Prevention, Liuzhou, China. 'Qingdao Center for Disease Control and Prevention, Qingdao, China. ${ }^{8}$ China National Center for Food Safety Risk Assessment, Beijing, China. ${ }^{9}$ The Copenhagen City Heart Study, Frederiksberg Hospital, Copenhagen University Hospital, Copenhagen, Denmark. ${ }^{10}$ Faculty of Health and Medical Sciences, University of Copenhagen, Copenhagen, Denmark.

${ }^{11}$ Peking University Institute of Environmental Medicine, Beijing, China.

Received: 24 April 2019 Accepted: 30 July 2019

Published online: 30 August 2019

\section{References}

1. Wang TJ, Pencina MJ, Booth SL, Jacques PF, Ingelsson E, Lanier K, et al. Vitamin D deficiency and risk of cardiovascular disease. Circulation. 2008; 117(4):503-11.

2. Brondum-Jacobsen P, Benn M, Jensen GB, Nordestgaard BG. 25hydroxyvitamin d levels and risk of ischemic heart disease, myocardial infarction, and early death: population-based study and meta-analyses of 18 and 17 studies. Arterioscler Thromb Vasc Biol. 2012;32(11):2794-802.

3. Al Mheid I, Quyyumi AA. Vitamin D and cardiovascular disease: controversy unresolved. J Am Coll Cardiol. 2017;70(1):89-100.

4. Chowdhury R, Kunutsor S, Vitezova A, Oliver-Williams C, Chowdhury S, Kiefte-de-Jong JC, et al. Vitamin D and risk of cause specific death: systematic review and meta-analysis of observational cohort and randomised intervention studies. BMJ. 2014;348:g1903.

5. Schottker B, Jorde R, Peasey A, Thorand B, Jansen EH, Groot L, et al. Vitamin $D$ and mortality: meta-analysis of individual participant data from a large consortium of cohort studies from Europe and the United States. BMJ. 2014;348:g3656.

6. Manson JE, Cook NR, Lee IM, Christen W, Bassuk SS, Mora S, et al. Vitamin D supplements and prevention of cancer and cardiovascular disease. New England J Med. 2019;380(1):33-44.

7. Kopecky SL, Bauer DC, Gulati M, Nieves JW, Singer AJ, Toth PP, et al. Lack of evidence linking calcium with or without vitamin $\mathrm{D}$ supplementation to cardiovascular disease in generally healthy adults: a clinical guideline from the National Osteoporosis Foundation and the American Society for Preventive Cardiology. Ann Intern Med. 2016;165(12):867-U189.

8. Wang L, Manson JE, Song Y, Sesso HD. Systematic review: vitamin D and calcium supplementation in prevention of cardiovascular events. Ann Intern Med. 2010;152(5):315-23.

9. Pilz S, Verheyen N, Grubler MR, Tomaschitz A, Marz W. Vitamin D and cardiovascular disease prevention. Nat Rev Cardiol. 2016;13(7):404-17.

10. Zittermann A, Ernst JB, Prokop S, Fuchs U, Dreier J, Kuhn J, et al. Effect of vitamin D on all-cause mortality in heart failure (EVITA): a 3-year randomized clinical trial with 4000 IU vitamin D daily. Eur Heart J. 2017;38(29):2279-86.

11. Bjelakovic G, Gluud LL, Nikolova D, Whitfield K, Wetterslev J, Simonetti RG, et al. Vitamin D supplementation for prevention of mortality in adults. Cochrane Db Syst Rev. 2014;10:(1):CD00747.

12. Brondum-Jacobsen $P$, Benn M, Afzal S, Nordestgaard BG. No evidence that genetically reduced 25-hydroxyvitamin $D$ is associated with increased risk of 
ischaemic heart disease or myocardial infarction: a Mendelian randomization study. Int J Epidemiol. 2015;44(2):651-61.

13. Afzal S, Brondum-Jacobsen P, Bojesen SE, Nordestgaard BG. Genetically low vitamin D concentrations and increased mortality: mendelian randomisation analysis in three large cohorts. BMJ. 2014;349:96330.

14. Lu L, Bennett DA, Millwood IY, Parish S, McCarthy MI, Mahajan A, et al. Association of vitamin D with risk of type 2 diabetes: a Mendelian randomisation study in European and Chinese adults. PLoS Med. 2018; 15(5):e1002566.

15. Lu L, Yu ZJ, Pan A, Hu FB, Franco OH, Li HX, et al. Plasma 25hydroxyvitamin $D$ concentration and metabolic syndrome among middle-aged and elderly Chinese individuals. Diabetes Care. 2009; 32(7):1278-83.

16. Demer LL, Hsu JJ, Tintut $Y$. Steroid hormone vitamin $D$ implications for cardiovascular disease. Circ Res. 2018;122(11):1576-85.

17. Benn $M$, Nordestgaard BG. From genome-wide association studies to Mendelian randomization: novel opportunities for understanding cardiovascular disease causality, pathogenesis, prevention, and treatment. Cardiovasc Res. 2018;114(9):1192-208.

18. Smith GD, Ebrahim S. 'Mendelian randomization': can genetic epidemiology contribute to understanding environmental determinants of disease? Int J Epidemiol. 2003;32(1):1-22.

19. Geng TT, Smith CE, Li CW, Huang T. Childhood BMI and adult type 2 diabetes, coronary artery diseases, chronic kidney disease, and cardiometabolic traits: a Mendelian randomization analysis. Diabetes Care. 2018;41(5):1089-96.

20. Huang $T$, Ding $M$, Bergholdt HKM, Wang TG, Heianza $Y$, Sun DJ, et al. Dairy consumption and body mass index among adults: Mendelian randomization analysis of 184802 individuals from 25 studies. Clin Chem. 2018;64(1):183-91.

21. Ding M, Huang T, Bergholdt HKM, Nordestgaard BG, Ellervik C, Qi L, et al. Dairy consumption, systolic blood pressure, and risk of hypertension: Mendelian randomization study. BMJ. 2017;356:j1000.

22. Wang TG, Huang T, Li YP, Zheng Y, Manson JE, Hu FB, et al. Low birthweight and risk of type 2 diabetes: a Mendelian randomisation study. Diabetologia. 2016;59(9):1920-7.

23. Ahn J, Yu K, Stolzenberg-Solomon R, Simon KC, McCullough ML, Gallicchio L, et al. Genome-wide association study of circulating vitamin D levels. Hum Mol Genet. 2010;19(13):2739-45.

24. Wang TJ, Zhang F, Richards JB, Kestenbaum B, van Meurs JB, Berry D, et al. Common genetic determinants of vitamin $D$ insufficiency: a genome-wide association study. Lancet. 2010;376(9736):180-8.

25. Thomsen $M$, Ingebrigtsen TS, Marott $J L$, Dahl M, Lange $P$, Vestbo J, et al. Inflammatory biomarkers and exacerbations in chronic obstructive pulmonary disease. Jama. 2013;309(22):2353-61.

26. Borenstein M, Hedges LV, Higgins JP, Rothstein HR. A basic introduction to fixed-effect and random-effects models for meta-analysis. Res Synth Methods. 2010;1(2):97-111.

27. Bowden J, Smith GD, Burgess S. Mendelian randomization with invalid instruments: effect estimation and bias detection through egger regression. Int J Epidemiol. 2015;44(2):512-25.

28. Tripkovic L, Lambert H, Hart K, Smith CP, Bucca G, Penson S, et al. Comparison of vitamin D2 and vitamin D3 supplementation in raising serum 25-hydroxyvitamin D status: a systematic review and meta-analysis. Am J Clin Nutr. 2012;95(6):1357-64.

29. Forouhi NG, Menon RK, Sharp SJ, Mannan N, Timms PM, Martineau $A R$, et al. Effects of vitamin D-2 or D-3 supplementation on glycaemic control and cardiometabolic risk among people at risk of type 2 diabetes: results of a randomized double-blind placebo-controlled trial. Diabetes Obes Metab. 2016;18(4):392-400.

30. Kuhn T, Kaaks R, Teucher B, Hirche F, Dierkes J, Weikert C, et al. Dietary, lifestyle, and genetic determinants of vitamin D status: a cross-sectional analysis from the European Prospective Investigation into Cancer and Nutrition (EPIC)-Germany study. Eur J Nutr. 2014;53(3):731-41.

31. Afzal S, Brondum-Jacobsen P, Bojesen SE, Nordestgaard BG. Vitamin D concentration, obesity, and risk of diabetes: a mendelian randomisation study. Lancet Diabetes Endocrinol. 2014;2(4):298-306.

32. Ooi EM, Afzal S, Nordestgaard BG. Elevated remnant cholesterol in 25hydroxyvitamin D deficiency in the general population: Mendelian randomization study. Circ Cardiovasc Genet. 2014;7(5):650-8.
33. Vimaleswaran KS, Berry DJ, Lu C, Tikkanen E, Pilz S, Hiraki LT, et al. Causal Relationship between obesity and vitamin D status: bi-directional Mendelian randomization analysis of multiple cohorts. Plos Med. 2013;10(2):e1001383.

34. Pascale AV, Finelli R, Giannotti R, Visco V, Fabbricatore D, Matula I, et al. Vitamin D, parathyroid hormone and cardiovascular risk: the good, the bad and the ugly. J Cardiovasc Med (Hagerstown). 2018;19(2):62-6.

35. Gambardella J, De Rosa M, Sorriento D, Prevete N, Fiordelisi A, Ciccarelli M, et al. Parathyroid hormone causes endothelial dysfunction by inducing mitochondrial ROS and specific oxidative signal transduction modifications. Oxidative Med Cell Longev. 2018;2018:9582319.

36. Afzal S, Bojesen SE, Nordestgaard BG. Low 25-hydroxyvitamin D and risk of type 2 diabetes: a prospective cohort study and metaanalysis. Clin Chem. 2013;59(2):381-91.

37. Vimaleswaran KS, Cavadino A, Berry DJ, Jorde R, Dieffenbach AK, Lu C, et al. Association of vitamin D status with arterial blood pressure and hypertension risk: a mendelian randomisation study. Lancet Diabetes Endo. 2014;2(9):719-29.

38. Barry EL, Peacock JL, Rees JR, Bostick RM, Robertson DJ, Bresalier RS, et al. Vitamin D receptor genotype, vitamin D3 supplementation, and risk of colorectal adenomas: a randomized clinical trial. JAMA Oncol. 2017; 3(5):628-35.

39. Grimnes G, Figenschau $Y$, Almas B, Jorde R. Vitamin D, insulin secretion, sensitivity, and lipids results from a case-control study and a randomized controlled trial using hyperglycemic clamp technique. Diabetes. 2011;60(11):2748-57.

40. Schwetz V, Scharnagl H, Trummer C, Stojakovic T, Pandis M, Grubler MR, et al. Vitamin D supplementation and lipoprotein metabolism: a randomized controlled trial. J Clin Lipidol. 2018;12(3):588-96.

41. Jorde R, Grimnes G. Vitamin D and metabolic health with special reference to the effect of vitamin D on serum lipids. Prog Lipid Res. 2011;50(4):303-12.

42. Jamilian M, Samimi M, Ebrahimi FA, Hashemi T, Taghizadeh M, Razavi M, et al. The effects of vitamin D and omega-3 fatty acid co-supplementation on glycemic control and lipid concentrations in patients with gestational diabetes. J Clin Lipidol. 2017;11(2):459-68.

43. Schnatz PF, Jiang XZ, Aragaki AK, Nudy M, O'Sullivan DM, Williams M, et al. Effects of calcium, vitamin $D$, and hormone therapy on cardiovascular disease risk factors in the Women's Health Initiative a randomized controlled trial. Obstet Gynecol. 2017;129(1):121-9.

44. Sollid ST, Hutchinson MYS, Fuskevag OM, Figenschau Y, Joakimsen RM, Schirmer $\mathrm{H}$, et al. No effect of high-dose vitamin D supplementation on glycemic status or cardiovascular risk factors in subjects with prediabetes. Diabetes Care. 2014;37(8):2123-31.

45. Verbanck M, Chen CY, Neale B, Do R. Detection of widespread horizontal pleiotropy in causal relationships inferred from Mendelian randomization between complex traits and diseases. Nat Genet. 2018;50(5):693.

46. Forouhi NG, Ye Z, Rickard AP, Khaw KT, Luben R, Langenberg C, et al. The association and causal inference between circulating 25-hydroxy vitamin D concentration and the risk of type 2 diabetes. Diabetologia. 2011;54:S75-S.

47. Scott RA, Scott LJ, Maegi R, Marullo L, Gaulton KJ, Kaakinen M, et al. An expanded genome-wide association study of type 2 diabetes in Europeans. Diabetes. 2017;66(11):2888-902.

\section{Publisher's Note}

Springer Nature remains neutral with regard to jurisdictional claims in published maps and institutional affiliations.

\section{Ready to submit your research? Choose BMC and benefit from:}

- fast, convenient online submission

- thorough peer review by experienced researchers in your field

- rapid publication on acceptance

- support for research data, including large and complex data types

- gold Open Access which fosters wider collaboration and increased citations

- maximum visibility for your research: over $100 \mathrm{M}$ website views per year

At $\mathrm{BMC}$, research is always in progress.

Learn more biomedcentral.com/submissions 